\title{
The Gogny-HFB+QRPA dipole strength function and its application to radia- tive neutron capture cross section
}

\author{
Stephane Goriely ${ }^{1, \star}$, Stephane Hilaire ${ }^{2,}$, and Sophie Péru², \\ ${ }^{1}$ Institut d'Astronomie et d'Astrophysique, Université Libre de Bruxelles, CP-226, 1050 Brussels, Belgium \\ ${ }^{2}$ CEA, DAM, DIF, F-91297 Arpajon, France
}

\begin{abstract}
Valuable theoretical predictions of nuclear dipole excitations in the whole chart are of great interest for different nuclear applications, including in particular nuclear astrophysics. Here we extend our large-scale calculations of the $E 1$ and $M 1$ absorption $\gamma$-ray strength function obtained in the framework of the axiallysymmetric deformed quasiparticle random phase approximation (QRPA) based on the finite-range D1M Gogny force to the determination of the de-excitation strength function. To do so, shell-model calculations of the de-excitation dipole strength function as well as experimental data are considered to provide insight in the low-energy limit and to complement the QRPA estimate phenomenologically. We compare our final prediction of the $E 1$ and $M 1$ strengths with available experimental data at low energies and show that a relatively good agreement can be obtained. Its impact on the average radiative width as well as radiative neutron capture cross section is discussed.
\end{abstract}

\section{Introduction}

Radiative neutron capture cross sections play a key role in almost all nuclear applications. Despite a huge effort to measure such radiative neutron capture cross sections, theoretical predictions are required to fill the gaps, both for nuclei for which measurements are not feasible at the present time, in particular for unstable targets, and for energies that cannot be reached in the laboratory. Some applications, such as nuclear astrophysics, also require the determination of radiative neutron capture cross sections for a large number of exotic neutron-rich nuclei [1]. In this case, large-scale calculations need to be performed on the basis of sound and accurate models to ensure a reliable extrapolation far away from the experimentally known region.

The neutron capture rates are commonly evaluated within the framework of the statistical model of HauserFeshbach, although the direct capture contribution plays an important role for very exotic nuclei [2]. The fundamental assumption of the Hauser-Feshbach model is that the capture goes through the intermediary formation of a compound nucleus in thermodynamic equilibrium. In this approach, the (n, $\gamma)$ cross section strongly depends on the electromagnetic interaction, i.e the photon de-excitation probability. In turn, it is well known that the photon strength function is dominated by the dipole contribution. The various multipolarities of the $\gamma$-ray strength function are traditionally modeled by the phenomenological Lorentzian approximation or some of its energy-dependent variants [3]. The reliability of the $\gamma$-ray strength predic-

\footnotetext{
^e-mail: sgoriely@astro.ulb.ac.be
}

tions can however be greatly improved by the use of microscopic or semi-microscopic models. Such an effort can be found in Refs. [4-8] where a complete set of $E 1$ and $M 1 \gamma$-ray strength functions was derived from mean field plus quasiparticle random phase approximation (QRPA) calculations. When compared with experimental data and considered for practical applications, all mean field plus QRPA calculations need however some phenomenological corrections. These include a broadening of the QRPA strength to take the neglected damping of collective motions into account as well as a shift of the strength to lower energies due to the contribution beyond the 1 particle 1 hole excitations and the interaction between the singleparticle and low-lying collective phonon degrees of freedom [9]. In addition, most of the mean field plus QRPA calculations assume spherical symmetries, so that phenomenological corrections need to be included in a way or another in order to properly describe the splitting of the giant dipole resonance in deformed nuclei [4-6].

Recently, axially-symmetric-deformed QRPA calculations based on Hartree-Fock-Bogoliubov (HFB) calculations using the finite-range Gogny interaction have been shown to provide rather satisfactory predictions of the $E 1$ [7] and $M 1$ strengths [8]. However, such QRPA calculations only describe the photoabsorption and it is well known that the de-excitation strength function may differ from the photoabsorption one, especially for low photon energies $[3,10]$. In particular, a non-zero limit of the dipole strength has been observed experimentally $[11,12]$ and confirmed by shell-model calculations [13-15].

In the present paper, we complement our previous HFB+QRPA study of the $E 1$ and $M 1$ strengths [7, 8], 
by correcting the low-energy limit on the basis of shellmodel calculation. The paper is organized as follows. In Sect. 2, the $E 1$ and $M 1 \mathrm{HFB}+\mathrm{QRPA}$ dipole photoabsorption strength functions are rapidly described together with the way corrections beyond QRPA are included phenomenologically. The de-excitation dipole strength function is discussed in Sect. 3 and low-energy corrections introduced to take into account the predictions of the shell model and available experimental data. In Sect 4, we compare measured average radiative width obtained with our D1M+QRPA strength and with Lorentzian-type models. Finally, the impact of the newly calculated $\gamma$-ray strength functions on the Hauser-Feshbach estimate of the radiative neutron capture cross section is discussed in Sect. 5. Conclusions are drawn in Sect. 6.

\section{E1 and M1 photoabsorption strength functions}

The QRPA formalism based on axially-symmetricdeformed HFB equations solved in a finite harmonic oscillator basis in cylindrical coordinates has been described in details in Refs. [7, 8, 16, 17]. In the present study, the D1M Gogny force [18] is used. Previous studies [7] have shown that the centroid energy of the $E 1$ strength is systematically larger by typically $2.5 \mathrm{MeV}$ than the Giant Dipole Resonance (GDR) energy determined experimentally. Similarly, a study of the QRPA low-energy vibrational states shows that the first experimental energies [19] are overestimated by typically $500 \mathrm{keV}$, as shown in Fig. 1 . For this reason, the effects beyond the 1 particle - 1 hole QRPA can be empirically included by considering an energy shift that increases with energy. In comparison with our previous study of the E1 QRPA strength [7], we have now adopted a simpler treatment of the renormalization procedure to reproduce both the experimental GDR properties and low-energy vibrational states. More specifically, for the $E 1$ and $M 1$ QRPA strengths, we now apply an energy shift of $\Delta=0.5 \mathrm{MeV}$ for $\varepsilon_{\gamma} \leq 0.5 \mathrm{MeV}, \Delta=2.5 \mathrm{MeV}$ for $\varepsilon_{\gamma}=18 \mathrm{MeV}$ and $\Delta=5 \mathrm{MeV}$ for $\varepsilon_{\gamma} \geq 21 \mathrm{MeV}$. For energies in the $0.5 \leq \varepsilon_{\gamma} \leq 21 \mathrm{MeV}$ range, the energy shift $\Delta$ is interpolated linearly between the anchor values at 0.5 , 18 and $21 \mathrm{MeV}$.

Similarly, an empirical damping of the collective motions is introduced in the QRPA strength by folding each $E 1$ strength by a standard Lorentzian (SLO) function of width $\Gamma$ that has been adjusted on photoabsorption data and is assumed to be dependent on the atomic mass $A$ only. We adopt the final expression $\Gamma[\mathrm{MeV}]=7-A / 45$ for $A \leq 200$, and $2.5 \mathrm{MeV}$ otherwise. For the $M 1$ strength, a constant value of $\Gamma=0.5 \mathrm{MeV}$ is adopted.

With such a systematic renormalization of the HFB+QRPA calculations, experimental strength functions based on photoabsorption measurements or nuclear resonance fluorescence can be rather well described, as already shown in Refs. [7, 8].
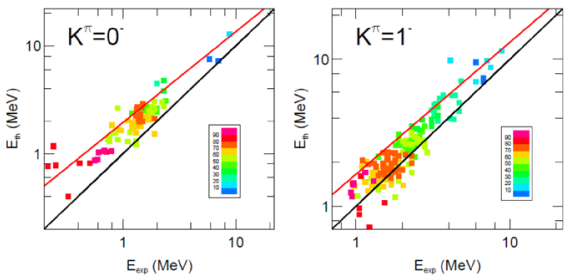

Figure 1. (Color online) Experimental - theoretical comparison of the energy of the low-energy vibrational states $K^{\pi}=0^{-}$(left panel) and $K^{\pi}=1^{-}$(right panel). The red curve corresponds to the energy shift applied to the HFB+QRPA strength. The colour code refers to the atomic mass range of the nuclei.

\section{E1 and M1 de-excitation strength functions}

When considering the de-excitation strength function, deviation from the photoabsorption strength can be expected, especially for $\gamma$-ray energies approaching the zero limit. In particular, a constant $E 1$ strength of $f_{E 1} \simeq 510^{-9} \mathrm{MeV}^{-3}$ at energies $\varepsilon_{\gamma} \lesssim 5 \mathrm{MeV}$ was found by the shell model [15]. In addition, shell-model calculations [13-15] predict an exponential increase of the $M 1$ de-excitation strength function at energies approaching zero. The upbend of the strength function observed experimentally $[11,12]$ has therefore been assumed to be of $M 1$ nature, though no experimental evidence exists for the moment. Therefore, the de-excitation strength function can be consistently taken from the QRPA calculation (Sect. 2) provided corrections at low energies inspired from shell model predictions are included. These finally are expressed as

$$
\begin{aligned}
& \overleftarrow{f_{E 1}}\left(\varepsilon_{\gamma}\right)=f_{E 1}^{Q R P A}\left(\varepsilon_{\gamma}\right)+f_{0} U /\left[1+e^{\left(\varepsilon_{\gamma}-\varepsilon_{0}\right)}\right] \\
& \overleftarrow{f_{M 1}}\left(\varepsilon_{\gamma}\right)=f_{M 1}^{Q R P A}\left(\varepsilon_{\gamma}\right)+C e^{-\eta \varepsilon_{\gamma}}\left[1+F \varepsilon_{\gamma}^{3}\right]
\end{aligned}
$$

where $f_{X 1}^{Q R P A}$ is the D1M+QRPA strength at the photon energy $\varepsilon_{\gamma}, U$ (in $\mathrm{MeV}$ ) is the excitation energy of the initial de-exciting state and $f_{0}=510^{-10} \mathrm{MeV}^{-4}$, $\varepsilon_{0}=5 \mathrm{MeV}, C=10^{-7} \mathrm{MeV}^{-3}, \eta=2 \mathrm{MeV}^{-1}$ and $F=0.4 \mathrm{MeV}^{-3}$ are free parameters that have been adjusted on the shell model results [15] and available experimental data $[11,12,21]$. The impact of the low-energy limit applied to the D1M+QRPA strength is illustrated in Fig. 2 for the $E 1$ channel in ${ }^{44} \mathrm{Ti}$ and in Fig. 3 for both the $E 1$ and $M 1$ contributions in ${ }^{57} \mathrm{Fe}$. The total dipole strength obtained with Eqs (1-2) is compared with experimental data extracted from photoabsorption measurements and the Oslo method in Fig. 4 for a sample of 4 nuclei. Note that the present $E 1$ and $M 1$ strengths are also found to be in rather good agreement with the data extracted from Average Resonance Capture (ARC) experiments, as shown in Ref. [20]. 


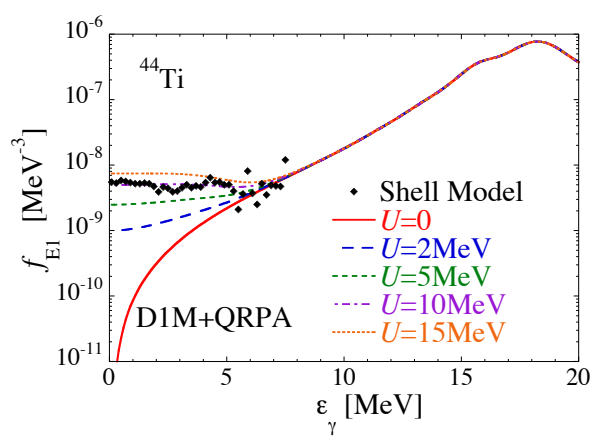

Figure 2. (Color online) Illustration for ${ }^{44} \mathrm{Ti}$ of the low-energy correction on the $E 1$ strength function (Eq. 1) applied to the $\mathrm{D} 1 \mathrm{M}+\mathrm{QRPA}$ strength for various initial excitation energies $U$, as suggested by the shell-model calculations [15].

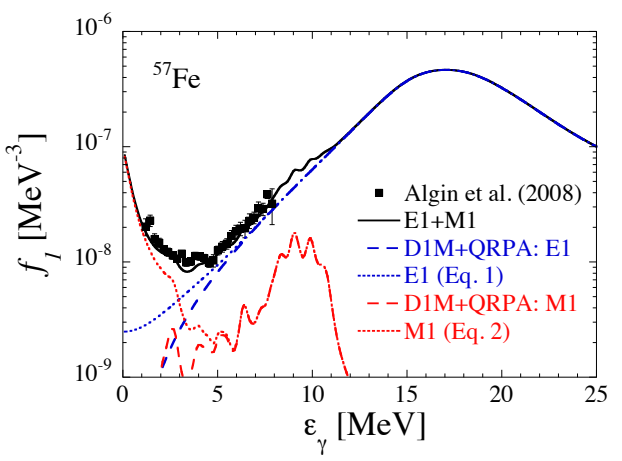

Figure 3. (Color online) Illustration for ${ }^{57} \mathrm{Fe}$ of the low-energy correction applied to the $E 1$ and $M 1 \mathrm{D} 1 \mathrm{M}+\mathrm{QRPA}$ strengths (Eqs. 1-2) and comparison with the low-energy data [21].

\section{Average radiative width}

Among the various experimental data, the average radiative width is known to play a key role in reaction modelling. The average radiative width is defined as [3]

$$
\left\langle\Gamma_{\gamma}\right\rangle=\frac{D_{0}}{2 \pi} \sum_{X, L, J, \pi} \int_{0}^{S_{n}+E_{n}} T_{X L}\left(\varepsilon_{\gamma}\right) \times \rho\left(S_{n}+E_{n}-\varepsilon_{\gamma}, J, \pi\right) d \varepsilon_{\gamma}
$$

where $D_{0}$ is the average resonance spacing for s-wave neutrons, $S_{n}$ is the neutron separation energy, $E_{n}$ is the neutron incident energy, $T_{X L}=2 \pi \varepsilon_{\gamma}^{2 L+1} \overleftarrow{f_{X L}}\left(\varepsilon_{\gamma}\right)$ is the electromagnetic transmission coefficient $(X=M$ or $E$ ) for a multipolarity $L$ and $\rho$ is the energy-, spin- and parity-dependent nuclear level density.

It has been a long-standing problem that phenomenological SLO models tend to overestimate the average ra-

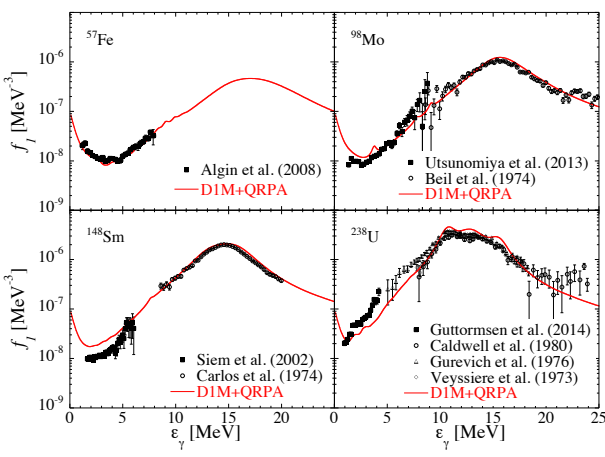

Figure 4. (Color online) The D1M+QRPA dipole strength function $f_{1}=f_{E 1}+f_{M 1}$ given by Eqs. (1-2) is compared with experimental photodata and Oslo measurements for ${ }^{57} \mathrm{Fe}[21],{ }^{98} \mathrm{Mo}$ $[22,23],{ }^{148} \mathrm{Sm}[24,25]$, and ${ }^{238} \mathrm{U}$ [26-29].

diative width significantly, while its improved and widely used version, the so-called Generalized Lorentzian (GLO) model [10], underestimates $\left\langle\Gamma_{\gamma}\right\rangle$. For this reason, the SLO model also tends to overestimate the radiative neutron capture cross sections for low-energy $(\mathrm{keV})$ neutrons, while the GLO model overestimates them (see also Ref. [30]). Such deviations are shown in Fig. 5 where the experimental average radiative widths are compared with predictions for SLO, GLO and the present corrected D1M+QRPA (Eqs. 1-2). It can be seen that in contrast to the SLO and GLO models, the present D1M+QRPA strengths (including both the $M 1$ and $E 1$ contributions) reproduce globally rather well the experimental average radiative width.

\section{Radiative neutron captures}

The radiative neutron capture cross sections and reaction rates of astrophysical interest have been calculated systematically on the basis of the Hauser-Feshbach statistical model described by the TALYS reaction code [31]. Both the widely used GLO model for the $E 1$ strength function and the present D1M+QRPA models corrected at low energies are considered here. The HFB plus combinatorial model of nuclear level densities [32] is adopted for the cross section calculations. Fig. 6 shows the ratio of the Maxwellian-averaged neutron capture cross sections (MACS) at a temperature $T=10^{9} \mathrm{~K}$ obtained with the present corrected D1M+QRPA to those obtained with the GLO $E 1$ model and SLO $M 1$ model recommended in Refs. [3, 10].

When approaching the neutron dripline $\left(S_{n}=0\right)$, the MACS calculated with the corrected D1M+QRPA strength is seen to be a factor 100 to 1000 larger than the one obtained with the traditional GLO model. The most significant effects responsible for such an increase of the MACS are $i$ ) the low-energy $E 1$ strength predicted for neutron-rich nuclei by the HFB+QRPA approach (as already discussed in Ref. [7]) and ii) the low-energy $M 1$ 


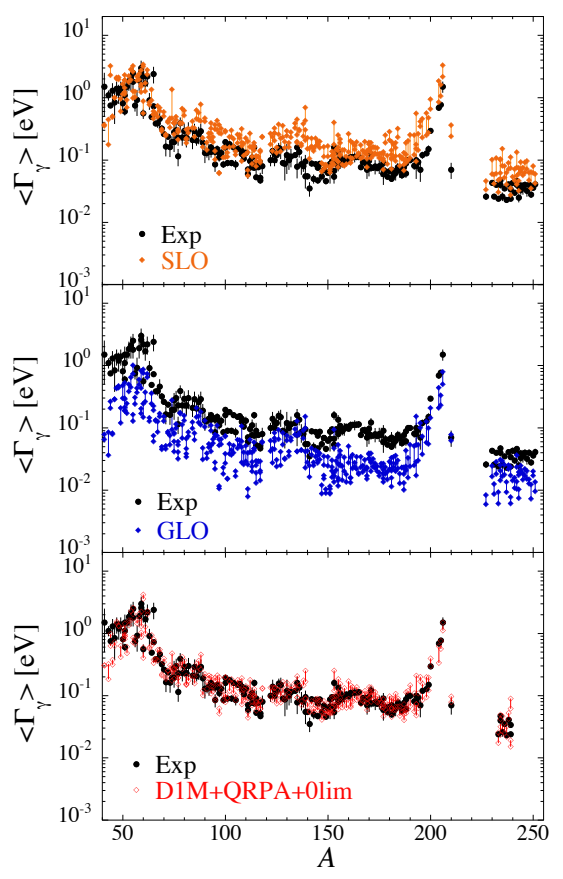

Figure 5. (Color online) Comparison between experimental (black circles) [3] and theoretical (coloured diamonds) average radiative width $\left\langle\Gamma_{\gamma}\right\rangle$ as a function of $A$. The upper panel is obtained with the SLO model [3] for both the $E 1$ and $M 1$ strengths and the middle panel with the GLO model for the $E 1$ and the SLO model for the $M 1[3,10]$. The lower panel corresponds to the present D1M+QRPA corrected by the low-energy limits (Eqs. 1-2). The error bars illustrate the uncertainties associated with the use of different nuclear level density models.

upbend introduced in Eq. (2). In particular, the $M 1$ upbend can affect the MACS of exotic neutron-rich nuclei by a factor up to 100 . In contrast, the low-energy limit of the $E 1$ strength function included in Eq. (1) only affects the MACS by 20 to $50 \%$ due to the $\varepsilon_{\gamma}^{3}$ dependence weighting the $\gamma$-ray strength function in the calculation of the transmission coefficient $T_{E 1}$ (see Sect. 4). This is not the case for the $M 1$ upbend, where the fast increase of the $M 1$ strength at decreasing energies approaching zero is capable of counterbalancing the $\varepsilon_{\gamma}^{3}$ effect in the calculation of $T_{M 1}$. As far as the HFB+QRPA prediction of the $M 1$ spin flip is concerned, it is almost always insignificant with respect to the stronger $E 1$ contribution at the relevant energies of $8-9 \mathrm{MeV}$, so that its impact on the MACS is reduced to typically $10 \%$ in comparison with the MACS obtained with a SLO $M 1$ strength [3]. However, the lowenergy $M 1$ scissors mode predicted in deformed nuclei by the HFB+QRPA can impact the MACS by a factor of 2 . This extra strength is never included in cross section cal-

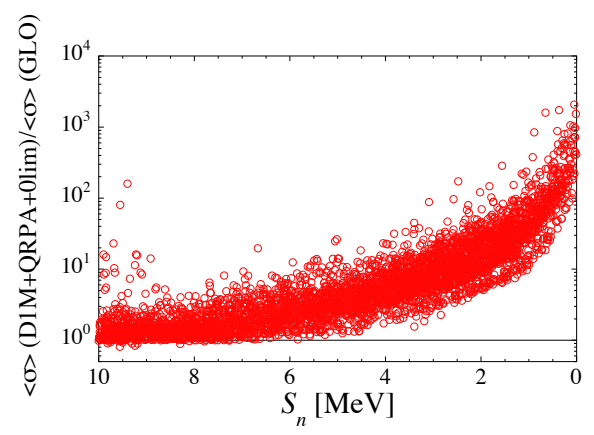

Figure 6. (Color online) Ratio of the MACS at $T=10^{9} \mathrm{~K} \mathrm{ob}-$ tained with the present D1M+QRPA corrected by the low-energy limits (Eqs. 1-2) to the one obtained with the GLO model for the $E 1$ and SLO model for the $M 1[3,10]$. The ratio is given for all nuclei with $8 \leq Z \leq 94$ lying between the proton and neutron driplines as a function of the neutron separation energy $S_{n}$.

culations except recently through a phenomenological approximation [30]. It affects the average radiative width as well as the neutron capture cross section of deformed nuclei in a non-negligible way.

\section{Conclusions}

HFB+QRPA models are now available for applications and have shown their capacity to predict photoabsorption $\gamma$-ray strength functions. Contributions beyond the 1 particle - 1 hole excitations and due to the interaction between the single-particle and low-lying collective phonon degrees of freedom can be empirically taken into account by renormalizing the HFB+QRPA strength through an energy shift and a damping width. Here we extend our $\mathrm{HFB}+\mathrm{QRPA}$ calculations to the determination of the deexcitation strength function. To do so, shell-model calculations of the de-excitation dipole strength function as well as experimental data are considered to provide insight in the low-energy limit. We showed that, in a first approximation, the HFB+QRPA strength can be complemented by simple analytical expressions to account for the missing strength at low energies. These corrections have been shown to affect the calculation of the average radiative width as well as radiative neutron capture cross sections, so that future work will need to estimate them more systematically on the basis of microscopic models. The present QRPA prediction provides an alternative accurate and reliable way to estimate the dipole $M 1$ and $E 1$ strength for a large set of nuclei with respect to the phenomenological and approximate Lorentzian-type description traditionally used in reaction codes.

Acknowledgments: We thank K. Sieja for valuable exchanges. SG acknowledges the support of the FRS-FNRS. 
This work was performed within the IAEA CRP on "Updating the Photonuclear data Library and generating a Reference Database for Photon Strength Functions" (F410 32).

\section{References}

[1] M. Arnould, S. Goriely, K. Takahashi, Phys. Repts. 450, 97 (2007)

[2] Y. Xu, S. Goriely, A. Koning, S. Hilaire, Phys. Rev. C 90, 024604 (2014)

[3] R. Capote, M. Herman, P. Oblozinsky, P. Young, S. Goriely, T. Belgya, A. Ignatyuk, A. Koning, S. Hilaire, V. Plujko et al., Nuclear Data Sheets 110, 3107 (2009)

[4] S. Goriely, E. Khan, Nucl. Phys. A 706, 217 (2002)

[5] S. Goriely, E. Khan, M. Samyn, Nucl. Phys. A 739, 331 (2004)

[6] I. Daoutidis, S. Goriely, Phys. Rev. C 86 (2012)

[7] M. Martini, S. Péru, S. Hilaire, S. Goriely, F. Lechaftois, Phys. Rev. C 94, 014304 (2016)

[8] S. Goriely, S. Hilaire, S. Péru, M. Martini, I. Deloncle, F. Lechaftois, Phys. Rev. C 94, 044306 (2016)

[9] S. Kamerdzhiev, V. Tkachev, Phys. Lett. B 142, 225 (1984)

[10] J. Kopecky, M. Uhl, Phys. Rev. C 41, 1941 (1990)

[11] A. Voinov, E. Algin, U. Agvaanluvsan, T. Belgya, R. Chankova, M. Guttormsen, G. Mitchell, J. Rekstad, A. Schiller, S. Siem, Phys. Rev. Lett. 93, 142504 (2004)

[12] M. Guttormsen, R. Chankova, U. Agvaanluvsan, E. Algin, L.A. Bernstein, F. Ingebretsen, T. Lönnroth, S. Messelt, G.E. Mitchell, J. Rekstad et al., Phys. Rev. C 71, 044307 (2005)

[13] R. Schwengner, S. Frauendorf, A.C. Larsen, Phys. Rev. Lett. 111, 232504 (2013)

[14] B.A. Brown, A.C. Larsen, Phys. Rev. Lett. 113 (2014)

[15] K. Sieja, Phys. Rev. Lett. 119, 052502 (2017)
[16] S. Peru, H. Goutte, Phys. Rev. C 77, 044313 (2008)

[17] S. Peru, M. Martini, Eur. Phys. J. A 50, 88 (2014)

[18] S. Goriely, S. Hilaire, M. Girod, S. Péru, Phys. Rev. Lett. 102, 242501 (2009)

[19] S. Hilaire, M. Girod, S. Goriely, A. Koning, Phys. Rev. C 86, 064317 (2012)

[20] J. Kopecky, S. Goriely, S. Peru, S. Hilaire, M. Martini, Phys. Rev. C 95, 054317 (2017)

[21] E. Algin, U. Agvaanluvsan, M. Guttormsen, A.C. Larsen, G.E. Mitchell, J. Rekstad, A. Schiller, S. Siem, A. Voinov, Phys. Rev. C 78, 054321 (2008)

[22] H. Utsunomiya, S. Goriely, T. Kondo, C. Iwamoto, H. Akimune, T. Yamagata, H. Toyokawa, H. Harada, F. Kitatani, Y.W. Lui et al., Physical review. C, Nuclear physics 88, 015805 (2013)

[23] H. Beil, R. Bergere, P. Carlos, A. Lepretre, A.D. Miniac, A. Veyssiere, Nucl. Phys. A 227, 427 (1974)

[24] S. Siem, M. Guttormsen, K. Ingeberg, E. Melby, J. Rekstad, A. Schiller, A. Voinov, Phys. Rev. C 65, 0044318 (2002)

[25] P. Carlos, H. Beil, R. Bergere, A. Lepretre, A.D. Miniac, A. Veyssière, Nucl. Phys. A 225, 171 (1974)

[26] M. Guttormsen, L.A. Bernstein, A. Görgen, B. Jurado, S. Siem, M. Aiche, Q. Ducasse, F. Giacoppo, F. Gunsing, T.W. Hagen et al., Phys. Rev. C 89, 014302 (2014)

[27] J.T. Caldwell, E.J. Dowdy, B.L. Berman, R.A. Alvarez, P. Meyer, Phys. Rev. C 21, 1215 (1980)

[28] G. Gurevich, L. Lazareva, V. Mazur, G. Solodukhov, B. Tulupov, Nucl. Phys. A 273, 326 (1976)

[29] A. Veyssiere, H. Beil, R. Bergere, P. Carlos, . Lepretre, K. Kernbath, Nucl. Phys. A 199, 45 (1973)

[30] M.R. Mumpower, T. Kawano, J.L. Ullmann, M. Krtička, T.M. Sprouse, Phys. Rev. C 96, 024612 (2017)

[31] A.J. Koning, D. Rochman, Nucl. Data Sheets 113, $2841(2012)$

[32] S. Goriely, S. Hilaire, A. Koning, Phys. Rev. C 78, 064307 (2008) 